\title{
Random Attractors for Stochastic Three-Component Reversible Gray-Scott System with Multiplicative White Noise
}

\author{
Anhui Gu' ${ }^{1,2}$ \\ ${ }^{1}$ Department of Mathematics, Shanghai Normal University, Shanghai 200234, China \\ ${ }^{2}$ College of Science, Guilin University of Technology, Guilin, Guangxi 541004, China \\ Correspondence should be addressed to Anhui Gu, gahui@glite.edu.cn
}

Received 25 December 2011; Revised 26 February 2012; Accepted 26 February 2012

Academic Editor: Oluwole D. Makinde

Copyright (c) 2012 Anhui Gu. This is an open access article distributed under the Creative Commons Attribution License, which permits unrestricted use, distribution, and reproduction in any medium, provided the original work is properly cited.

The paper is devoted to proving the existence of a compact random attractor for the random dynamical system generated by stochastic three-component reversible Gray-Scott system with multiplicative white noise.

\section{Introduction}

Let $\mathcal{O}$ be an open bounded set of $\mathbf{R}^{n}(n \leq 3)$ with a locally Lipschitz continuous boundary $\partial \mathcal{O}$. We consider the stochastic three-component reversible Gray-Scott system with multiplicative noise

$$
\begin{gathered}
\frac{\partial \tilde{u}}{\partial t}=d_{1} \Delta \tilde{u}-(F+k) \tilde{u}+\tilde{u}^{2} \widetilde{v}-G \tilde{u}^{3}+N \tilde{w}+\sigma \tilde{u} \circ \frac{d B_{t}}{d t}, \\
\frac{\partial \widetilde{v}}{\partial t}=d_{2} \Delta \widetilde{v}+F(1-\tilde{v})-\tilde{u}^{2} \widetilde{v}+G \tilde{u}^{3}+\sigma \widetilde{v} \circ \frac{d B_{t}}{d t}, \\
\frac{\partial \tilde{w}}{\partial t}=d_{3} \Delta \tilde{w}+k \tilde{u}-(F+N) \tilde{w}+\sigma \tilde{w} \circ \frac{d B_{t}}{d t},
\end{gathered}
$$

where $\tilde{u}=\widetilde{u}(x, t), \widetilde{v}=\widetilde{v}(x, t)$, and $\widetilde{w}=\widetilde{w}(x, t)$ are real-valued functions on $\mathcal{O} \times[\tau,+\infty)$, $\tau \in \mathbf{R}$; all the parameters are arbitrarily given positive constants; $B_{t}$ is a Brownian motion 
and $\circ$ denotes the Stratonovich sense of the stochastic term. In this work, we consider the homogenous Neumann boundary condition

$$
\frac{\partial \tilde{u}}{\partial v}(x, t)=\frac{\partial \widetilde{v}}{\partial v}(x, t)=\frac{\partial \widetilde{w}}{\partial v}(x, t)=0, \quad x \in \partial \mathcal{O},
$$

where $\partial / \partial \boldsymbol{v}$ is the outward normal derivative, and with an initial condition

$$
\tilde{u}(x, 0)=\tilde{u}_{0}(x), \quad \tilde{v}(x, 0)=\widetilde{v}_{0}(x), \quad \widetilde{w}(x, 0)=\widetilde{w}_{0}(x), \quad x \in \mathcal{O} .
$$

The three-component reversible Gray-Scott model was firstly introduced by Mahara et al. [1]. Recently in [2], You gave the existence of global attractor for system (1.1) when $\sigma=0$ with Neumann boundary condition (1.2) on a bounded domain of space dimension $n \leq 3$ by the method of the rescaling and grouping estimate. However, the reactions and diffusions are often affected by stochastic factors then it is important and meaningful to take the asymptotic behavior of solutions to consideration. Particularly, the dynamics of certain systems frequently follows some self-organization process where the development of new, complex structures takes place primarily in and through the system itself. This self-organization is normally triggered by internal variation processes, which are usually called fluctuations or noise, that have a positive influence on the system. For instance, recent theoretical studies and experiments with cultured glial cells and the Belousov-Zhabotinsky reaction have shown that noise may play a constructive role on the dynamical behavior of spatially extended systems [3-5]. Therefore, one cannot ignore the role of noise in chemical and biological self-organization and its relationship with the environmental selection of emergent patterns [6]. In [7-9], the influence of additive noise on Gray-Scott systems was discussed. As pointed in $[10,11]$, the effects of additive and multiplicative noises are fundamentally different in nonlinear systems. While the effect of additive noise does not depend on the state of the system, the effect of multiplicative noise is state dependent. Natural systems in which the effect of noise on the system's dynamics does depend on the recent state are autocatalytic chemical reactions or growth processes in developmental biology. More generally speaking, in each system whose dynamics shows some degree of selfreferentiality, the effect of exogenous noise will depend on the recent system's state. If noise is multiplicative, "new" phenomena can occur; that is, the noisy system can exhibit behavior, which is qualitatively different from that of the deterministic system, a phenomenon that has been coined noise-induced transitions.

A fundamental problem in the study of dynamics of a stochastic partial differential equation is to show that it generates a random dynamical system (or stochastic flow). One of the most interesting concepts of the theory of random dynamical systems is the random attractor, which was introduced in the 90s of the last century (see [12]). An attractor for an autonomous dynamical system is a compact set in the phase space, attracting the image of particular sets of initial states under the evolution of the dynamical system. However, the random case is more complicated, because random attractors depend on the random parameter and have their own temporal dynamics induced by the noise (cf. the definition in Section 3). Moreover, the existence of a random attractor to the stochastic reversible GrayScott system, especially of three components, is widely open to the best of our knowledge. According to methodology of [2] of nondissipative coupling of three variables and the coefficients barrier, we consider system (1.1)-(1.3), which gives partly an answer to the 
problems of random perturbations proposed in [13]. In this paper, we use the notions and frameworks in $[12,14,15]$ to study the stochastic three-component reversible Gray-Scott system with multiplicative white noise.

The paper is organized as follows. In Section 2, we give the existence and uniqueness of solution. Section 3 is devoted to the existence of a random attractor.

\section{Existence and Uniqueness of Solutions}

Let $(\Omega, \mathcal{F}, P)$ be a probability space, and $\left\{\theta_{t}: \Omega \rightarrow \Omega, t \in \mathbf{R}\right\}$ is a family of measure preserving transformations such that $(t, \omega) \mapsto \theta_{t} \omega$ is measurable, $\theta_{0}=i d$, and $\theta_{t+s}=\theta_{t} \theta_{s}$ for all $s, t \in \mathbf{R}$. The flow $\theta_{t}$ together with the probability space $\left(\Omega, \mathcal{F}, P,\left(\theta_{t}\right)_{t \in \mathbf{R}}\right)$ is called as a measurable dynamical system.

A random dynamical system (RDS) on a Polish space $(X, d)$ with Borel $\sigma$-algebra $B$ over $\theta$ on $(\Omega, \mathcal{F}, P)$ is a measurable map

$$
\varphi: \mathbf{R}^{+} \times \Omega \times X \longrightarrow X, \quad(t, \omega, x) \longmapsto \varphi(t, \omega) x,
$$

such that $P$-almost surely $(P$-a.s. $)$ we have

(i) $\varphi(0, \omega)=i d$ on $X$;

(ii) (cocycle property) $\varphi(t+s, \omega)=\varphi\left(t, \theta_{s} \omega\right) \varphi(s, \omega)$ for all $s, t \geq 0$.

An RDS is continuous or differentiable if $\varphi(t, \omega): X \rightarrow X$ is continuous or differentiable.

A map $B: \Omega \rightarrow 2^{X}$ is said to be a closed (compact) random set if $B(\omega)$ is closed (compact) for $P$-a.s. $\omega \in \Omega$ and if $\omega \mapsto d(x, B(\omega))$ is $P$-a.s. measurable for all $x \in X$.

Consider the product Hilbert spaces $L^{2}(\mathcal{O}), H=\left[L^{2}(\mathcal{O})\right]^{3}$, and $H^{1}(\mathcal{O}), E=\left[H^{1}(\mathcal{O})\right]^{3}$ with the usual inner products and norms

$$
\begin{gathered}
(u, v)=\int_{\Omega} u v d x, \quad|u|=(u, u)^{1 / 2} \quad \forall u, v \in L^{2}(\mathcal{O}) \\
((u, v))=\sum_{i=1}^{n}\left(D_{i} u, D_{i} v\right)+F(u, v), \quad\|u\|=((u, u))^{1 / 2} \quad \forall u, v \in H^{1}(\mathcal{O}) .
\end{gathered}
$$

Obviously, for fixed $F$ the scalar product and norm defined above are equivalent to the usual scalar product and norm in $H^{1}(\mathcal{O})$. And the norm of $L^{p}(\mathcal{O})$ will be denoted by $|\cdot|_{L^{p}}$ if $p \neq 2, X=\left(L^{6}(\mathcal{O})\right)^{3}$.

Define the unbounded positive linear operator

$$
A(D(A) \longrightarrow H)=\tilde{\Delta}-F I,
$$


where

$$
\begin{gathered}
\tilde{\Delta}=\left(\begin{array}{ccc}
d_{1} \Delta & 0 & 0 \\
0 & d_{2} \Delta & 0 \\
0 & 0 & d_{3} \Delta
\end{array}\right), \\
D(A)=\left\{(\phi, \varphi, \psi) \in\left[H^{2}(\mathcal{O})\right]^{3}: \frac{\partial \phi}{\partial \boldsymbol{v}}=\frac{\partial \varphi}{\partial \boldsymbol{v}}=\frac{\partial \psi}{\partial \boldsymbol{v}}=0 \text { on } \partial \mathcal{O}\right\} .
\end{gathered}
$$

By the Lumer-Phillips theorem and the generation theorem for analytic semigroup [16], the operator $A$ in (2.3) is linear, sectorial, closed, and defined and is the generator of an analytic $C_{0}$-semigroup $\left\{e^{A t}, t \geq 0\right\}$ on the Hilbert space $H$. Its spectral set consists of only nonnegative eigenvalues, denoted by $\mu_{i}=\lambda_{i}+F, i \geq 0$, where $\lambda_{i}$ are the corresponding eigenvalues of $\tilde{\Delta}$ satisfying

$$
0=\lambda_{0}<\lambda_{1} \leq \lambda_{2} \leq \cdots \leq \lambda_{i} \leq \cdots \quad\left(\lambda_{i} \longrightarrow \infty \text { as } i \longrightarrow \infty\right)
$$

By the fact that $H^{1}(\mathcal{O}) \hookrightarrow L^{6}(\mathcal{O})$ is a continuous embedding for $n \leq 3$ and by the generalized Hölder inequality, one has

$$
\left|u^{2} v\right| \leq|u|_{L^{6}}^{2}|v|_{L^{6}}, \quad \text { for } u, v \in L^{6}(\mathcal{O})
$$

Therefore, the nonlinear mapping defined on $E$,

$$
f(\tilde{u}, \widetilde{v}, \tilde{w})=\left(\begin{array}{c}
-k \tilde{u}+\tilde{u}^{2} \tilde{v}-G \tilde{u}^{3}+N \tilde{w} \\
F-\tilde{u}^{2} \widetilde{v}+G \tilde{u}^{3} \\
k \tilde{u}-N \tilde{w}
\end{array}\right): E \longrightarrow H
$$

is locally Lipschitz continuous. Thus, the initial boundary problem (1.1)-(1.3) is formulated as an initial value problem of the stochastic three-component reversible Gray-Scott system with multiplicative noise

$$
\frac{d \widetilde{g}}{d t}=A \widetilde{g}+f(\widetilde{g})+\sigma \widetilde{g} \circ \frac{d B_{t}}{d t}
$$

and an initial condition

$$
\tilde{g}(0)=\widetilde{g}_{0}=\left(\widetilde{u}_{0}, \widetilde{v}_{0}, \widetilde{w}_{0}\right) \in H,
$$

where $\tilde{g}(t)=(\tilde{u}(t, \cdot), \tilde{v}(t, \cdot), \tilde{w}(t, \cdot)) . B_{t}$ is a one-dimensional two-sided Wiener process on a probability space $(\Omega, \mathcal{F}, P)$, where

$$
\Omega=\left\{\omega \in C\left(\mathbf{R}, \mathbf{R}^{m}\right): \omega(0)=0\right\},
$$


the Borel $\sigma$-algebra $\mathcal{F}$ on $\Omega$ is generated by the compact open topology, and $P$ is the corresponding Wiener measure on $\mathcal{F}$. We can define a family of measure-preserving and ergodic transformations (a flow) $\left\{\theta_{t}\right\}_{t \in \mathbf{R}}$ by

$$
\theta_{t} \omega(\cdot)=\omega(\cdot+t)-\omega(t)
$$

By means of the change of variables

$$
\begin{aligned}
u(t) & =\alpha(t) \tilde{u}(t), \\
v(t) & =\alpha(t) \tilde{v}(t) \quad \text { with } \alpha(t)=e^{-\sigma B_{t}}, \\
w(t) & =\alpha(t) \tilde{w}(t),
\end{aligned}
$$

system (1.1) can be written as

$$
\begin{gathered}
\frac{\partial u}{\partial t}=d_{1} \Delta u-(F+k) u+\alpha^{-2}(t) u^{2} v-G \alpha^{-2}(t) u^{3}+N w \\
\frac{\partial v}{\partial t}=d_{2} \Delta v+F(\alpha(t)-v)-\alpha^{-2}(t) u^{2} v+G \alpha^{-2}(t) u^{3} \\
\frac{\partial w}{\partial t}=d_{3} \Delta w+k u-(F+N) w
\end{gathered}
$$

That is $g(t, \cdot)=(u(t, \cdot), v(t, \cdot), w(t, \cdot))$ satisfies

$$
\frac{d g}{d t}=A g+\tilde{f}(g, \omega)
$$

with initial condition

$$
g(0)=\tilde{g}(0)=g_{0}=\left(u_{0}, v_{0}, w_{0}\right) \in H,
$$

where

$$
\tilde{f}(g, w)=\left(\begin{array}{c}
-k u+\alpha^{-2}(t) u^{2} v-G \alpha^{-2}(t) u^{3}+N w \\
F-\alpha^{-2}(t) u^{2} v+G \alpha^{-2}(t) u^{3} \\
k u-N w
\end{array}\right)
$$

Due to the fact that $H^{1}(\mathcal{O}) \hookrightarrow L^{6}(\mathcal{O})$ and $(2.6)$, we know that $\tilde{f}(g, \omega)$ is locally Lipschitz continuous with respect to $g$ and bounded for every $\omega \in \Omega$. By the same method in [17, Chapters II and XV], we can prove for $P$-a.s. every $\omega \in \Omega$ the local existence and uniqueness of the weak solution $g(t), t \in[\tau, T]$ for some $\tau<T$, of (2.16) with $g(\tau)=g_{0}$, which is continuously depending on the initial data $g_{0} \in H$ and turns out to be a strong solution 
on $(\tau, T]$ by [16, Theorem 48.5]. One can show that for $P$-a.s. every $\omega \in \Omega$, the following statements hold for all $\tau<T$.

(i) If $\tilde{g}(0, \omega) \in H$, then $\tilde{g}(t, \omega)$ lies in

$$
C([\tau, T) ; H) \cap C^{1}((\tau, T) ; H) \cap L^{2}([\tau, T) ; E) .
$$

(ii) $\tilde{g}(t, \tilde{g}(0, \omega))$ is jointly continuous in $t$ and $\tilde{g}(0, \omega)$ in $[\tau, T) \times H$.

(iii) The solution mapping of (2.16) satisfies the property of an RDS.

This system has a unique solution for every $\omega \in \Omega$. Hence the solution mapping

$$
\widetilde{S}(t, \omega): \widetilde{g}(\tau, \omega) \longmapsto \widetilde{g}(t, \omega)
$$

generates an RDS. So the transformation

$$
\widehat{S}(t, \omega): \alpha^{-1}(t) \tilde{g}(\tau, \omega) \longmapsto \alpha^{-1}(t) \tilde{g}(t, \omega)
$$

also determines an RDS corresponding to system (1.1).

We will prove the existence of a nonempty compact random attractor for the RDS $\widehat{S}(t, \omega)$.

\section{Existence of a Random Attractor}

A random set $\mathcal{K}(\omega)$ is said to absorb the set $B \subset X$ for an $\operatorname{RDS} \varphi$ if $P$-a.s. there exists $t_{B}(\omega)$ such that

$$
\varphi\left(t, \theta_{-t} \omega\right) B \subset \mathcal{K}(\omega) \quad \forall t \geq t_{B}(\omega)
$$

A random set $\mathcal{A}(\omega)$ is said to be a random attractor associated to the $\operatorname{RDS} \varphi$ if $P$-a.s.:

(i) $\mathcal{A}(\omega)$ is a random compact set, that is, $P$-a.s. $\omega \in \Omega, \mathcal{A}(\omega)$ is compact, and for all $x \in X$ and $P$-a.s. the map $x \mapsto \operatorname{dist}(x, \mathcal{A}(\omega))$ is measurable.

(ii) $\varphi(t, \omega) \mathcal{A}(\omega)=A\left(\theta_{t} \omega\right)$ for all $t \geq 0$ (invariance).

(iii) For all bounded $B \subset X$,

$$
\lim _{t \rightarrow \infty} \operatorname{dist}\left(\varphi\left(t, \theta_{-t} \omega\right) B, \mathcal{A}(\omega)\right)=0,
$$

where $\operatorname{dist}(\cdot, \cdot)$ denotes the Hausdorff semidistance:

$$
\operatorname{dist}(Y, Z)=\sup _{x \in Y} \inf d(x, y), \quad Y, Z \in X
$$


Proposition 3.1 (see $[14,15]$ ). Let $\phi$ be an RDS on a Polish space $(X, d)$ with Borel $\sigma$-algebra B over the flow $\left\{\theta_{t}\right\}_{t \in \mathbf{R}}$ on a probability space $(\Omega, \mathcal{F}, P)$. Suppose there exists a random compact set $\mathcal{K}(\omega)$ such that for any bounded nonrandom set $B \subset X P$-a.s

$$
\operatorname{dist}\left(\varphi\left(t, \theta_{-t} \omega\right) B, \mathcal{K}(\omega)\right) \longrightarrow 0 \quad \text { as } t \longrightarrow+\infty
$$

Then the set

$$
\mathcal{A}(\omega)=\overline{\bigcup_{B \subset X} \Lambda_{B}(\omega)}
$$

is a unique random attractor for $\phi$, where the union is taken over all bounded $B \subset X$ and $\Lambda_{B}(\omega)$ is the omega-limit set of $B$ given by

$$
\Lambda_{B}(\omega)=\bigcap_{s \geq 0} \overline{\bigcup_{t \geq s} \phi\left(t, \theta_{-t} \omega\right) B}
$$

Now, we will show the existence of a random attractor for the RDS (2.16).

Lemma 3.2. There exists a random variable $r_{1}(\omega)>0$, depending on $F, G, \sigma$, and $\mu$, such that for all $\rho>0$ there exists $t(\omega) \leq-1$ such that the following holds $P$-a.s. For all $t_{0} \leq t(\omega)$, and for all $\tilde{g}_{0} \in H$ with $\left|\widetilde{g}_{0}\right| \leq \rho$, the solution $g\left(t, \omega ; t_{0}, \alpha\left(t_{0}\right) \widetilde{g}_{0}\right)$ of system $(2.16)$ over $\left[t_{0}, \infty\right)$, with $g\left(t_{0}\right)=\alpha\left(t_{0}, \omega\right) \widetilde{g}_{0}$, satisfies the inequality

$$
\left|g\left(-1, \omega ; t_{0}, \alpha\left(t_{0}, \omega\right) \tilde{g}_{0}\right)\right|^{2} \leq r_{1}^{2}(\omega)
$$

Proof. Define

$$
W(t, x)=\frac{N}{k} w(t, x), \quad \mu=\frac{k}{N} .
$$

Then (2.13)-(2.15) can be written as

$$
\begin{gathered}
\frac{\partial u}{\partial t}=d_{1} \Delta u-(F+k) u+\alpha^{-2}(t) u^{2} v-G \alpha^{-2}(t) u^{3}+k W, \\
\frac{\partial v}{\partial t}=d_{2} \Delta v+F(\alpha(t)-v)-\alpha^{-2}(t) u^{2} v+G \alpha^{-2}(t) u^{3}, \\
\mu \frac{\partial W}{\partial t}=\mu d_{3} \Delta W+k u-(\mu F+k) W .
\end{gathered}
$$


Taking the inner products of (3.9)-(3.11) with $G u(t), v(t)$, and $G W(t)$, respectively. Then sum up the resulting equalities. By the Neumann boundary condition (1.2), we get

$$
\begin{aligned}
& \frac{1}{2} \frac{d}{d t}\left(G|u|^{2}+|v|^{2}+\mu G|W|^{2}\right)+G(F+k)|u|^{2}+F|v|^{2}+G(\mu F+k)|W|^{2}+d_{1} G\|u\|^{2}+d_{2}\|v\|^{2}+\mu G d_{3}\|W\|^{2} \\
& \quad \leq \int_{\Omega} F \alpha(t) v d x+2 k G \int_{\Omega} u W d x-\alpha^{-2}(t) \int_{\Omega}\left(G u^{2}-u v\right)^{2} d x \\
& \quad \leq k G|u|^{2}+k G|W|^{2}+\frac{F}{2}|v|^{2}+\frac{F \alpha^{2}(t)}{2}|\mathcal{O}|
\end{aligned}
$$

where $|\mathcal{O}|$ denotes the volume of $\mathcal{O}$. Set

$$
d=\min \left\{d_{1}, d_{2}, d_{3}\right\}, \quad C_{1}=\frac{F|\mathcal{O}|}{\min \{1, G, G / \mu\}} .
$$

Then (3.12) yields

$$
\frac{d}{d t}|g(t)|^{2}+d\|g(t)\|^{2}+F|g(t)|^{2} \leq C_{1} \alpha^{2}(t)
$$

Applying Gronwall's inequality to (3.14) and then integrating in $\left[t_{0},-1\right]$, with $t_{0} \leq-1$ we have

$$
\begin{aligned}
|g(-1)|^{2} & \leq\left|\alpha\left(t_{0}\right) g_{0}\right|^{2} e^{-F\left(-1-t_{0}\right)}+C_{1} \int_{t_{0}}^{-1} e^{-F(-1-s)} \alpha^{2}(s) d s \\
& \leq e^{F}\left(e^{F t_{0}}\left|\alpha\left(t_{0}\right) g_{0}\right|^{2}+C_{1} \int_{t_{0}}^{-1} e^{F s} \alpha^{2}(s) d s\right) .
\end{aligned}
$$

Consequently, give $B(0, \rho) \subset H, P$-a.s. there exists $t(\omega, \rho) \leq-1$ such that for $t_{0} \leq t(\omega, \rho)$ and all $g_{0} \in B(0, \rho)$,

$$
\left|g\left(-1, \omega ; t_{0}, \alpha\left(t_{0}\right) \widetilde{g}_{0}\right)\right|^{2} \leq r_{1}^{2}(\omega)
$$

with

$$
r_{1}^{2}(\omega)=e^{F}\left(1+C_{1} \int_{-\infty}^{-1} e^{F s} \alpha^{2}(s) d s\right)
$$

Indeed, it is enough to choose $t(\omega, \rho)$ such that

$$
e^{F t_{0}} \alpha^{2}\left(t_{0}\right) \rho^{2} \leq 1
$$

and take into account (3.15) and the fact that P-a.s. $e^{F s} \alpha^{2}(s)=e^{F s} e^{-2 \sigma W_{s}} \rightarrow 0$ as $s \rightarrow-\infty$. 
If we now return to (3.14) and integrate for $t \in[-1,0]$, we have

$$
\begin{gathered}
|g(t)|^{2} \leq|g(-1)|^{2} e^{-F(t+1)}+C_{1} \int_{-1}^{t} e^{-F(t-s)} \alpha^{2}(s) d s, \\
d \int_{-1}^{0}\|g(s)\|^{2} d s \leq|g(-1)|^{2}+C_{1} \int_{-1}^{0} \alpha^{2}(s) d s .
\end{gathered}
$$

Thus, we can conclude that given $B(0, \rho) \subset H$ and $P$-a.s. there exists $t(\omega, \rho) \leq-1$ such that for $t_{0} \leq t(\omega, \rho)$ and for all $g_{0} \in B(0, \rho)$,

$$
\begin{gathered}
\left|g\left(t, \omega ; t_{0}, g_{0}\right)\right|^{2} \leq e^{-F(t+1)} r_{1}^{2}(\omega)+C_{1} \int_{-1}^{t} e^{-F(t-s)} \alpha^{2}(s) d s, \\
d \int_{-1}^{0}\left\|g\left(s, \omega ; t_{0}, g_{0}\right)\right\|^{2} d s \leq r_{1}^{2}(\omega)+C_{1} \int_{-1}^{0} \alpha^{2}(s) d s .
\end{gathered}
$$

To prove the absorption at time $t=0$, we need the following proposition.

Proposition 3.3. There exists a random variable $r_{3}(\omega)>0$, depending on $\lambda_{1}, \sigma$, and $d$, such that for all $\rho^{\prime}>0$ there exists $t(\omega) \leq-1$ such that the following holds $P$-a.s. For all $t_{0} \leq t(\omega)$ and for all $\widetilde{g}_{0} \in H$ with $\left|\widetilde{g}_{0}\right| \leq \rho^{\prime}$, the solution $g\left(t, \omega ; t_{0}, \alpha\left(t_{0}\right) g_{0}\right)$ of system $(2.16)$ over $\left[t_{0}, \infty\right)$, with $g\left(t_{0}\right)=\alpha\left(t_{0}, \omega\right) \tilde{g}_{0}$, satisfies the inequality

$$
\left|g\left(-1, \omega ; t_{0}, \alpha\left(t_{0}, \omega\right) \tilde{g}_{0}\right)\right|_{X}^{6} \leq r_{3}^{2}(\omega)
$$

Also, for $t \in[-1,0]$ one has

$$
|g(t)|_{X}^{6} \leq e^{-F(t+1)} r_{3}^{2}(\omega)+C_{4} \int_{-1}^{t} e^{-F(t-\tau)} \alpha^{6}(\tau) d \tau
$$

Proof. Letting $V(t, x)=v(t, x) / G,(3.9)-(3.11)$ can be written as

$$
\begin{gathered}
\frac{\partial u}{\partial t}=d_{1} \Delta u-(F+k) u+G \alpha^{-2}(t) u^{2} V-G \alpha^{-2}(t) u^{3}+k W \\
\frac{\partial V}{\partial t}=d_{2} \Delta v+\frac{F}{G} \alpha(t)-F V-\alpha^{-2}(t) u^{2} V+\alpha^{-2}(t) u^{3} \\
\mu \frac{\partial W}{\partial t}=\mu d_{3} \Delta W+k u-(\mu F+k) W .
\end{gathered}
$$


Take the inner products $\left((3.23), u^{5}(t)\right),\left((3.24), G V^{5}(t)\right)$ and $\left((3.25), W^{5}(t)\right)$ and sum up the resulting equalities. By the Neumann boundary condition, we get

$$
\begin{aligned}
\frac{1}{6} \frac{d}{d t}\left(|u|_{L^{6}}^{6}+G|V|_{L^{6}}^{6}+\mu|W|_{L^{6}}^{6}\right)+5\left(d_{1}\left\|u^{2} \nabla u\right\|^{2}+d_{2} G\left\|V^{2} \nabla V\right\|^{2}+\mu d_{3} G\left\|W^{2} \nabla W\right\|^{2}\right) \\
=-(F+k) \int_{\Omega} u^{6} d s+F \int_{\Omega} \alpha(t) V^{5} d x-G F \int_{\Omega} V^{6} d x \\
-(\mu F+k) \int_{\Omega} W^{6} d x+k \int_{\Omega} u^{5} W d x+k \int_{\Omega} u W^{5} d x \\
-G \alpha^{-2}(t) \int_{\Omega}\left(u^{8}-u^{7} V-u^{3} V^{5}+u^{2} V^{6}\right) d x .
\end{aligned}
$$

By using Young's inequality, we obtain

$$
\begin{aligned}
& -G \alpha^{-2}(t) \int_{\Omega}\left(u^{8}-u^{7} V-u^{3} V^{5}+u^{2} V^{6}\right) d x \leq 0 \\
& k \int_{\Omega} u^{5} W d x+k \int_{\Omega} u W^{5} d x \leq k|u|_{L^{6}}^{6}+k|W|_{L^{6}}^{6} .
\end{aligned}
$$

From (3.27), (3.26) yields

$$
\frac{d}{d t}\left(|u|_{L^{6}}^{6}+G|V|_{L^{6}}^{6}+\mu|W|_{L^{6}}^{6}\right)+F\left(|u|_{L^{6}}^{6}+G|V|_{L^{6}}^{6}+\mu|W|_{L^{6}}^{6}\right) \leq \frac{F|\mathcal{O}|}{G^{5}} \alpha^{6}(t),
$$

that is,

$$
\frac{d}{d t}\left(|u|_{L^{6}}^{6}+G^{-5}|v|_{L^{6}}^{6}+\mu^{-5}|w|_{L^{6}}^{6}\right)+F\left(|u|_{L^{6}}^{6}+G^{-5}|v|_{L^{6}}^{6}+\mu^{-5}|w|_{L^{6}}^{6}\right) \leq \frac{F|\mathcal{O}|}{G^{5}} \alpha^{6}(t) .
$$

By denoting

$$
C_{4}=\frac{F|\mathcal{O}|}{G^{5} \min \left\{1, G^{-5}, \mu^{-5}\right\}}
$$

then (3.29) implies that

$$
\frac{d}{d t}|g(t)|_{X}^{6}+F|g(t)|_{X}^{6} \leq C_{4} \alpha^{6}(t)
$$

Integrating in $\left[t_{0},-1\right]$, with $t_{0} \leq-1$ we have

$$
|g(-1)|_{X}^{6} \leq\left|\alpha\left(t_{0}\right) g_{0}\right|_{X}^{6} e^{-F\left(-1-t_{0}\right)}+C_{4} \int_{t_{0}}^{-1} e^{-F(-1-s)} \alpha^{6}(s) d s .
$$


Consequently, given $B\left(0, \rho^{\prime}\right) \subset H, P$-a.s. there exists $t\left(\omega, \rho^{\prime}\right) \leq-1$ such that for all $t_{0} \leq t\left(\omega, \rho^{\prime}\right)$ and for all $g_{0} \in B\left(0, \rho^{\prime}\right)$,

$$
\left|g\left(-1, \omega, t_{0}, \alpha\left(t_{0}\right) g_{0}\right)\right|_{X}^{6} \leq r_{3}^{2}(\omega)
$$

with

$$
r_{3}^{2}(\omega)=e^{F}\left(1+C_{4} \int_{-\infty}^{-1} e^{F s} \alpha^{6}(s) d s\right)
$$

In fact, it is enough to choose $t\left(\omega, \rho^{\prime}\right)$ to satisfy

$$
e^{F t_{0}} \alpha^{6}\left(t_{0}\right) \rho^{\prime^{6}} \leq 1
$$

and take into (3.32) and the fact that $P$-a.s. $e^{F s} \alpha^{6}(s)=e^{F s} e^{-6 \sigma W_{s}} \rightarrow 0$ as $s \rightarrow-\infty$. Also, from (3.32) and for $t \in[-1,0]$ we get

$$
|g(t)|_{X}^{6} \leq e^{-F(t+1)} r_{3}^{2}(\omega)+C_{4} \int_{-1}^{t} e^{-F(t-\tau)} \alpha^{6}(\tau) d \tau .
$$

Lemma 3.4. There exists a random variable $r_{2}(\omega)>0$, depending on $F, G, N, d, k$, and $\sigma$, such that for all $\rho>0$ there exists $t(\omega) \leq-1$ such that the following holds $P$-a.s. For all $t_{0} \leq t(\omega)$ and for all $\widetilde{g}_{0} \in H$ with $\left|\tilde{g}_{0}\right| \leq \rho$, there exists a unique solution $g\left(t, \omega ; t_{0}, \alpha\left(t_{0}\right) g_{0}\right)$ of system $(2.16)$ over $\left[t_{0}, \infty\right)$, with $g\left(t_{0}\right)=\alpha\left(t_{0}, \omega\right) \tilde{g}_{0}$, and put $\tilde{g}\left(t, \omega ; t_{0}, \widetilde{g}_{0}\right):=\alpha^{-1}(t, \omega) g\left(t, \omega ; t_{0}, \widetilde{g}_{0}\right)$. Then

$$
\left\|\widetilde{g}\left(0, \omega ; t_{0}, \tilde{g}_{0}\right)\right\|^{2} \leq r_{2}^{2}(\omega)
$$

Proof. To get a bound in $E$, we multiply (2.13)-(2.15) by $-\Delta u,-\Delta v$, and $--\Delta w$, respectively. Add up the three equalities, and due to the Neumann boundary condition, we have

$$
\begin{aligned}
\frac{1}{2} \frac{d}{d t}( & \left.\|u\|^{2}+\|v\|^{2}+\|w\|^{2}\right)+d_{1}|\Delta u|^{2}+d_{2}|\Delta v|^{2}+d_{3}|\Delta w|^{2}+(F+k)\|u\|^{2}+F\|v\|^{2}+(F+N)\|w\|^{2} \\
= & -\alpha^{-2}(t) \int_{\Omega} u^{2} v \Delta u d x+G \alpha^{-2}(t) \int_{\Omega} u^{3} \Delta u d x-N \int_{\Omega} w \Delta u d x \\
& -F \alpha(t) \int_{\Omega} \Delta v d x+\alpha^{-2}(t) \int_{\Omega} u^{2} v \Delta v d x-G \alpha^{-2}(t) \int_{\Omega} u^{3} \Delta v d x \\
& -k \int_{\Omega} u \Delta w d x \\
\leq & \frac{d_{1}}{2}|\Delta u|^{2}+\frac{d_{2}}{2}|\Delta v|^{2}+\frac{d_{3}}{2}|\Delta w|^{2}+\alpha^{-4}(t)\left(\frac{1}{d_{1}}+\frac{1}{d_{2}}\right) \int_{\Omega} u^{4} v^{2} d x \\
& +\frac{N^{2}}{d_{1}} \int_{\Omega} w^{2} d x+\frac{k^{2}}{2 d_{3}} \int_{\Omega} u^{2} d x+\frac{G^{2} \alpha^{-4}(t)}{d_{2}} \int_{\Omega} u^{6} d x
\end{aligned}
$$


that is,

$$
\begin{aligned}
& \frac{d}{d t}\left(\|u\|^{2}+\|v\|^{2}+\|w\|^{2}\right)+F\left(\|u\|^{2}+\|v\|^{2}+\|w\|^{2}\right) \\
& \quad \leq \alpha^{-4}(t)\left(\frac{4}{3 d_{1}}+\frac{4}{3 d_{2}}+\frac{2 G^{2}}{d_{2}}\right) \int_{\Omega}\left(u^{6}+v^{6}\right) d x+\frac{2 N^{2}}{d_{1}}|w|^{2}+\frac{k^{2}}{d_{3}}|u|^{2} \\
& \quad \leq C_{2} \alpha^{-4}(t)|g|_{X}^{6}+C_{3}|g|^{2} .
\end{aligned}
$$

Here $d=\min \left\{d_{1}, d_{2}, d_{3}\right\}, C_{2}=(1 / d)\left(8 / 3+2 G^{2}\right), C_{3}=(1 / d) \max \left\{2 N^{2}, k^{2}\right\}$. Then from (3.39) we have

$$
\frac{d}{d t}\|g\|^{2}+F\|g\|^{2} \leq C_{2} \alpha^{-4}(t)|g|_{X}^{6}+C_{3}|g|^{2}
$$

Integrating (3.40) in $[s, 0], s \in[-1,0]$, we obtain

$$
\|g(0)\|^{2} \leq\|g(s)\|^{2}+C_{2} \int_{s}^{0} \alpha^{-4}(\tau)|g(\tau)|_{X}^{6} d \tau+C_{3} \int_{s}^{0}|g(\tau)|^{2} d \tau
$$

Integrating (3.41) in $[-1,0]$ and by (3.20),

$$
\begin{aligned}
\|g(0)\|^{2} & \leq \int_{-1}^{0}\|g(\tau)\|^{2} d \tau+C_{2} \int_{-1}^{0} \alpha^{-4}(\tau)|g(\tau)|_{X}^{6} d \tau+C_{3} \int_{-1}^{0}|g(\tau)|^{2} d \tau \\
& \leq\left(\frac{1}{d}+\frac{C_{3}}{F}\right)\left(r_{1}^{2}(\omega)+C_{1} \int_{-1}^{0} \alpha^{2}(\tau) d \tau\right)+C_{2} \sup _{-1 \leq t \leq 0} \alpha^{-4}(t) \int_{-1}^{0}|g(\tau)|_{X}^{6} .
\end{aligned}
$$

It is now straightforward from (3.31) and (3.36) that

$$
\begin{aligned}
\|g(0)\|^{2}=\|\tilde{g}(0)\|^{2} \leq & \left(\frac{1}{d}+\frac{C_{3}}{F}\right)\left(r_{1}^{2}(\omega)+C_{1} \int_{-1}^{0} \alpha^{2}(\tau) d \tau\right) \\
& +\frac{C_{2}}{F} \sup _{-1 \leq t \leq 0} \alpha^{-4}(t)\left(r_{3}^{2}(\omega)+C_{4} \int_{-1}^{0} \alpha^{6}(\tau) d \tau\right) .
\end{aligned}
$$

Consequently, $P$-a.s. there exists $r^{2}(\omega)$ such that given $\rho>0$, there exists $\widetilde{t}(\omega) \leq-1$ such that for all $t_{0} \leq \tilde{t}(\omega)$ and $\widetilde{g}_{0} \in H$ with $\left|\widetilde{g}_{0}\right| \leq \rho$,

$$
\left\|\tilde{g}\left(0, \omega ; t_{0}, \tilde{g}_{0}\right)\right\|^{2} \leq r_{2}^{2}(\omega),
$$


where

$$
\begin{aligned}
r_{2}^{2}(\omega)= & \left(C_{5}+C_{6}\right) e^{F}+C_{1} C_{5} \int_{-\infty}^{-1} e^{F(s+1)} \alpha^{2}(s) d s+C_{1} C_{5} \int_{-1}^{0} \alpha^{2}(s) d s \\
& +C_{4} C_{6} \int_{-\infty}^{-1} e^{F(s+1)} \alpha^{6}(s) d s+C_{4} C_{6} \int_{-1}^{0} \alpha^{6}(s) d s, \\
C_{5} & =\left(\frac{1}{d}+\frac{C_{3}}{F}\right), \quad C_{6}=\frac{C_{2}}{F} \sup _{-1 \leq t \leq 0} \alpha^{-4}(t) .
\end{aligned}
$$

Thus, we can have the main result.

Theorem 3.5. The RDS $\widehat{S}(t, \omega)$ has a nonempty compact random attractor $\mathcal{A}(\omega)$.

Proof. This follows from Lemma 3.2 and Lemma 3.4 combined with the embedding of $E \hookrightarrow H$ and Proposition 3.1.

Remark 3.6. It is necessary and interesting for us to consider the Hausdorff dimension of the random attractor which is generated by the stochastic three-component reversible Gray-Scott system with multiplicative white noise, but it seems impossibile to apply the results in $[18,19]$ directly because of the higher-order terms. In order to verify the differentiability properties for the cocycle generated by the random system, we need to check condition (2.7)-(2.13) in [19]. Considering the linearized equation (2.13)-(2.15), we have

$$
\begin{gathered}
\frac{\partial \tilde{U}}{\partial t}=d_{1} \Delta \tilde{U}-(F+k) \tilde{U}+2 u v e^{2 \sigma W_{t}} \tilde{U}+u^{2} \tilde{V} e^{2 \sigma W_{t}}-3 G e^{2 \sigma W_{t}} u^{2} \tilde{U}+N \widetilde{W} \\
\frac{\partial \tilde{V}}{\partial t}=d_{1} \Delta \tilde{V}-F \tilde{V}-2 u v e^{2 \sigma W_{t}} \tilde{U}-u^{2} \tilde{V} e^{2 \sigma W_{t}}+3 G u^{2} e^{2 \sigma W_{t}} \tilde{U} \\
\frac{\partial \widetilde{W}}{\partial t}=d_{1} \Delta \widetilde{W}+k \tilde{U}-(F+N) \widetilde{W} .
\end{gathered}
$$

Letting $\Phi(t)=(\tilde{U}, \tilde{V}, \widetilde{W}), q(t)=\tilde{g}(t)-\bar{g}(t)-\Phi(t)$, then

$$
q(t)=\left(q_{1}(t), q_{2}(t), q_{3}(t)\right)=(\tilde{u}-\bar{u}-\tilde{U}, \tilde{v}-\bar{v}-\tilde{V}, \tilde{w}-\bar{w}-\widetilde{W})
$$

satisfies

$$
\begin{aligned}
\frac{\partial q_{1}}{\partial t}= & d_{1} \triangle q_{1}-(F+k) q_{1}+\alpha(t)\left(\tilde{u}^{2} \tilde{v}-\bar{u}^{2} \bar{v}\right)-2 \tilde{u} \tilde{v} e^{2 \sigma W_{t}}(t) \tilde{U}-\tilde{u}^{2} e^{2 \sigma W_{t}} \tilde{V} \\
& +3 G \tilde{u}^{2} e^{2 \sigma W_{t}} \tilde{U}-G \alpha(t)\left(\tilde{u}^{3}-\bar{u}^{3}\right)+N q_{3}, \\
\frac{\partial q_{2}}{\partial t}= & d_{1} \triangle q_{2}-F q_{2}-\alpha(t)\left(\tilde{u}^{2} \tilde{v}-\bar{u}^{2} \bar{v}\right)+2 \tilde{u} \tilde{v} e^{2 \sigma W_{t}} \tilde{U}+\tilde{u}^{2} e^{2 \sigma W_{t}} \tilde{V} \\
& -3 G \tilde{u}^{2} e^{2 \sigma W_{t}} \tilde{U}+G \alpha(t)\left(\tilde{u}^{3}-\bar{u}^{3}\right), \\
\frac{\partial q_{3}}{\partial t}= & d_{1} \triangle q_{3}+k q_{1}-(F+N) q_{3} .
\end{aligned}
$$


Here, let

$$
\begin{aligned}
& \tilde{g}(t)=\tilde{g}\left(t, \omega ; 0, \widetilde{g}_{0}\right)=\left(\tilde{u}\left(t, \omega ; 0, \tilde{u}_{0}\right), \widetilde{v}\left(t, \omega ; 0, \widetilde{v}_{0}\right), \tilde{w}\left(t, \omega ; 0, \tilde{w}_{0}\right)\right) \\
& \bar{g}(t)=\bar{g}\left(t, \omega ; 0, \bar{g}_{0}\right)=\left(\bar{u}\left(t, \omega ; 0, \bar{u}_{0}\right), \bar{v}\left(t, \omega ; 0, \bar{v}_{0}\right), \bar{w}\left(t, \omega ; 0, \bar{w}_{0}\right)\right)
\end{aligned}
$$

be two solutions of system (1.1). From (3.48), it seems hard to get the conclusion.

\section{Acknowledgments}

The authors would like to thank the referees for many helpful suggestions and comments. Also more thanks to Professor Shengfan Zhou and Professor Yuncheng You for their helpful discussions, advice, and assistance. This work was supported by the National Natural Science Foundation of China under Grant 11071165 and Guangxi Provincial Department of Research Project under Grant 201010LX166.

\section{References}

[1] H. Mahara, N. J. Suematsu, T. Yamaguchi, K. Ohgane, Y. Nishiura, and M. Shimomura, “Three-variable reversible Gray-Scott model," Journal of Chemical Physics, vol. 121, no. 18, pp. 8968-8972, 2004.

[2] Y. You, "Dynamics of three-component reversible Gray-Scott model," Discrete and Continuous Dynamical Systems, vol. 14, no. 4, pp. 1671-1688, 2010.

[3] P. Jung, A. Cornell-Bell, F. Moss, S. Kadar, J. Wang, and K. Showalter, "Noise sustained waves in subexcitable media: from chemical waves to brain waves," Chaos, vol. 8, no. 3, article 567, 9 pages, 1998.

[4] J. Sancho and J. Garcia-Ojalvo, "Noise-induced order in extended systems: a tutorial," in Stochastic Processes in Physics, Chemistry and Biology, J. A. Freund and T. Pschel, Eds., vol. 557 of Lecture Notes in Physics, pp. 235-246, Springer, 2000.

[5] J. Wang, S. Kádár, P. Jung, and K. Showalter, "Noise driven avalanche behavior in subexcitable media," Physical Review Letters, vol. 82, no. 4, pp. 855-858, 1999.

[6] G. Nicolis and I. Prigogine, Self-Organization in Nonequilibrium Systems. From Dissipative Structures to Order through Fluctuations, John Wiley \& Sons, New York, NY, USA, 1977.

[7] Y. Hayase and H. R. Brand, "The Gray-Scott model under the influence of noise: reentrant spatiotemporal intermittency in a reaction-diffusion system," Journal of Chemical Physics, vol. 123, no. 12, Article ID 124507, 5 pages, 2005.

[8] D. Hochberg, F. Lesmes, F. Morán, and J. Pérez-Mercader, "Large-scale emergent properties of an autocatalytic reaction-diffusion model subject to noise," Physical Review E, vol. 68, no. 6, Article ID 066114, 10 pages, 2003.

[9] M. P. Zorzano, D. Hochberg, and F. Morán, “Dynamic renormalization group and noise induced transitions in a reaction diffusion model," Physica A, vol. 334, no. 1-2, pp. 67-77, 2004.

[10] W. Horsthemke and R. Lefever, Noise-induced Transitions: Theory and Applications in Physics, Chemistry, and Biology, vol. 15, Springer, Berlin, Germany, 1984.

[11] A. Schenzle and H. Brand, "Multiplicative stochastic processes in statistical physics," Physics Letters A, vol. 69, no. 5, pp. 313-315, 1979.

[12] L. Arnold, Random Dynamical Systems, Springer, Berlin, Germany, 1998.

[13] Y. You, "Dynamics of two-compartment Gray-Scott equations," Nonlinear Analysis: Theory, Methods $\mathcal{E}$ Applications, vol. 74, no. 5, pp. 1969-1986, 2011.

[14] I. Chueshov, Monotone Random Systems Theory and Applications, Springer, New York, NY, USA, 2002.

[15] T. Caraballo, J. A. Langa, and J. C. Robinson, "Stability and random attractors for a reaction-diffusion equation with multiplicative noise," Discrete and Continuous Dynamical Systems, vol. 6, no. 4, pp. 875892, 2000.

[16] G. R. Sell and Y. You, Dynamics of Evolutionary Equations, vol. 143, Springer, New York, NY, USA, 2002.

[17] V. V. Chepyzhov and M. I. Vishik, Attractors for Equations of Mathematical Physics, vol. 49, American Mathematical Society, Providence, RI, USA, 2002. 
[18] A. Debussche, "On the finite dimensionality of random attractors," Stochastic Analysis and Applications, vol. 15, no. 4, pp. 473-491, 1997.

[19] A. Debussche, "Hausdorff dimension of a random invariant set," Journal de Mathématiques Pures et Appliquées, vol. 77, no. 10, pp. 967-988, 1998. 


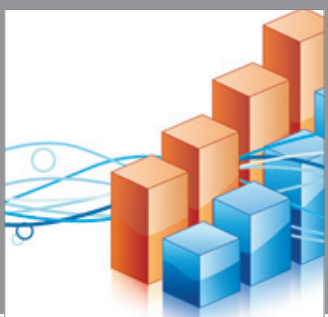

Advances in

Operations Research

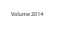

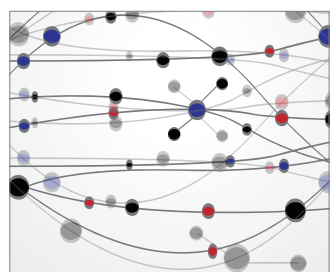

\section{The Scientific} World Journal
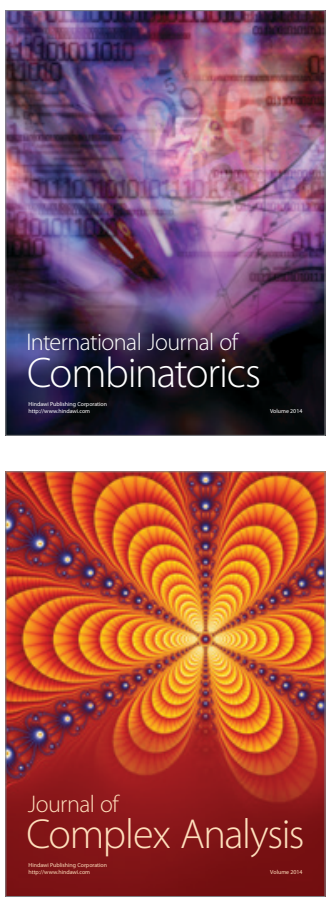

International Journal of

Mathematics and

Mathematical

Sciences
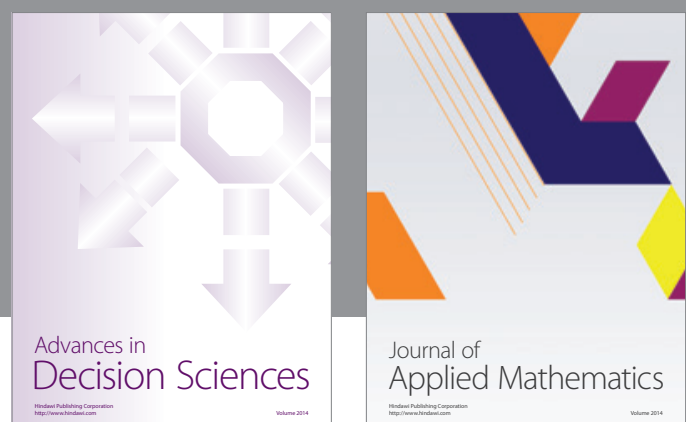

Journal of

Applied Mathematics
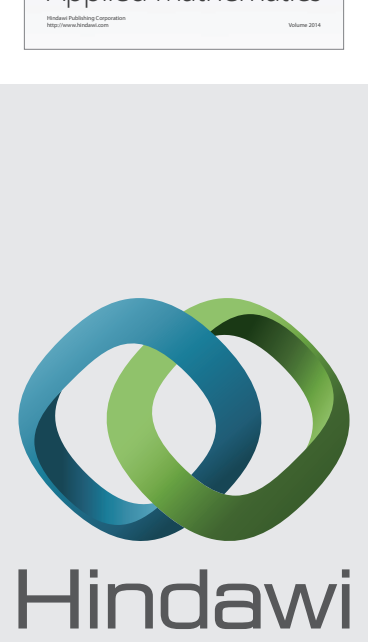

Submit your manuscripts at http://www.hindawi.com
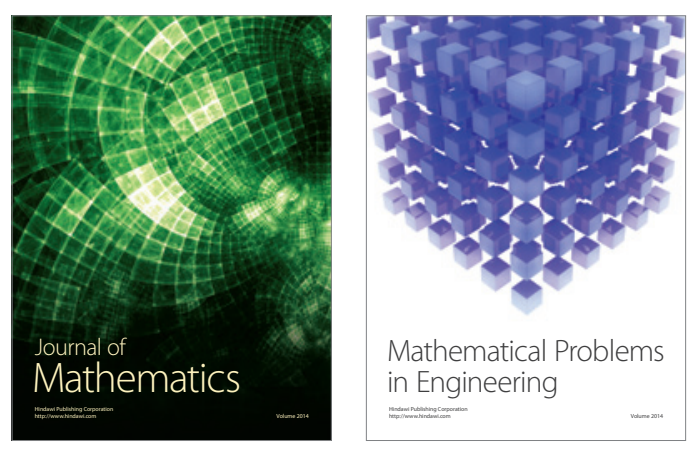

Mathematical Problems in Engineering
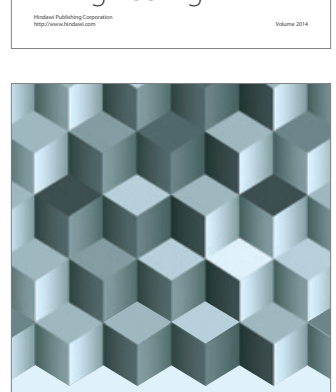

Journal of

Function Spaces
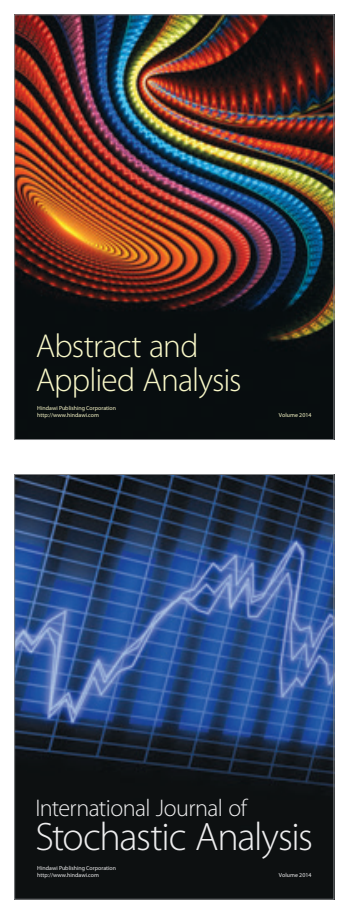

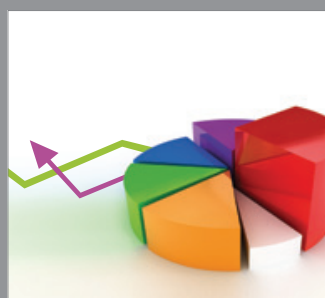

ournal of

Probability and Statistics

Promensencen
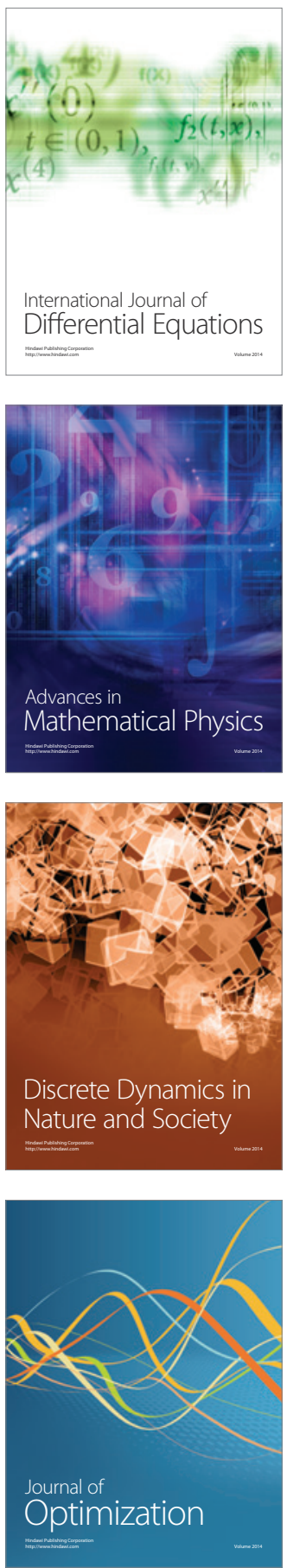